\title{
Self-reported reasons for treatment nonadherence in chronic obstructive pulmonary disease (COPD) patients: a 24-week prospective cohort study in China
}

\author{
Mingzhou Zhang", Ting Tang", Min Wan, Qiao Zhang, Changzheng Wang, Qianli Ma \\ Institute of Respiratory Disease, Xinqiao Hospital, Third Military Medical University, Chongqing, China \\ Contributions: (I) Conception and design: Q Ma, C Wang, Q Zhang; (II) Administrative support: C Wang, Q Ma; (III) Provision of study materials or \\ patients: Q Zhang, M Wan, M Zhang, T Tang; (IV) Collection and assembly of data: Q Ma, Q Zhang, M Wan; (V) Data analysis and interpretation: \\ Q Ma, Q Zhang, M Zhang, T Tang; (VI) Manuscript writing: All authors; (VII) Final approval of manuscript: All authors. \\ \#These authors contributed equally to this work. \\ Correspondence to: Qianli Ma. Institute of Respiratory Disease, Xinqiao Hospital, Third Military Medical University, Chongqing, China. \\ Email: cqmq1@163.com.
}

\begin{abstract}
Background: Adherence to medication plays an important role in the control of disease progression and treatment costs for patients with chronic obstructive pulmonary disease (COPD). Existing studies are mostly retrospective studies based on local medical insurance databases and prescription records and therefore cannot analyze or determine the causes of poor adherence in COPD patients.

Methods: This is a prospective study that enrolled 1,223 COPD patients, including 929 newly diagnosed patients and 294 previously diagnosed patients. Telephone follow-up was conducted to administer a questionnaire on treatment adherence and collect detailed information on the prescriptions and the actual use of medications for COPD. The follow-up period was 24 weeks.

Results: COPD patients had adherence rates of $74.65 \%, 49.63 \%$, and $33.61 \%$ at $4-$, $12-$, and $24-$ week telephone follow-ups, respectively. Cox regression showed that women, newly diagnosed patients, patients with a mild Global Initiative for Chronic Obstructive Lung Disease (GOLD) stage, and patients with fewer routes of administration had poorer adherence. According to the subgroup analysis of the diagnostic status, the overall adherence of the newly diagnosed patients was poorer than that of the previously diagnosed patients. Among the causes of nonadherence, drug stoppage after remission (27.2\%) was the most common cause, followed by economic factors $(21.5 \%)$ and poor efficacy $(21.1 \%)$.

Conclusions: This is the first study focusing on the adherence behavior of newly diagnosed COPD patients and the first large-scale clinical study on the Chinese population. Overall, sex, diagnostic status (newly diagnosed and previously diagnosed), GOLD stage, and routes of administration were the four major factors that affected the adherence of COPD patients.
\end{abstract}

Keywords: Chronic obstructive pulmonary disease (COPD); adherence; newly diagnosed patient

Submitted Jul 16, 2020. Accepted for publication Sep 16, 2020.

doi: $10.21037 /$ apm-20-1741

View this article at: http://dx.doi.org/10.21037/apm-20-1741

\section{Introduction}

Chronic obstructive pulmonary disease (COPD) is one of the leading causes of disability and death in the world, and the number of COPD patients in China exceeds 100 million (1). COPD is a chronic disease. Although it cannot be cured, effective management can control its symptoms, delay its progression, and improve the quality of life $(2,3)$. The Global Initiative for Chronic Obstructive Lung Disease (GOLD) recommends continuous treatment with long-acting drugs for most COPD patients (1). Less 
Box 1 Causes of non-adherence

High cost of medication

Diminished treatment effectiveness with long-term use

Poor self-efficacy

Relief of symptoms after short-term controller medication use

Occurrence/fear of side effects

Difficulty obtaining medication

Inconvenience of treatment

Preference for oral medication

Other

medication adherence is associated with more severe COPD symptoms, increased hospitalization rates, greater mortality, and higher treatment cost (4). In addition, a study in the United States found that compared with patients with poor prescription adherence, patients with good prescription adherence had fewer hospitalizations and lower medical expenses (5). Therefore, effective medication management for COPD can not only improve the clinical symptoms and prognosis of individual patients but also reduce the family's and society's medical expenses. However, in real life, it has been found that COPD patients have low adherence to medication, especially to long-term medication. For example, studies on treatment with longacting beta-agonists in Germany and the United States showed that the nonpersistence rate of treatment with longacting $\beta 2$ agonists, which are commonly used for COPD, was up to $65-70 \%(6,7)$. Therefore, understanding the main causes of nonadherence to medication and giving targeted interventions on this basis may improve the outcome of COPD treatment.

The causes of the nonadherence to medication are complex. Increasing evidence indicates that the invariable factors include sex, economic environment, and cultural background. These factors can help identify patients with a high risk of nonadherence at the initial diagnosis. The variable factors, such as individual beliefs regarding health and illness, are also correlated with medication nonadherence $(8,9)$, and the identification of these factors is conducive to the early adoption of targeted interventions in clinical practice.

Many previous studies are based on a retrospective analysis of local medical insurance databases and prescription records, so they lack data that can reflect the causes of nonadherence. Some patients never have a followup visit after staring treatment, whose information is absent in these retrospective studies. Furthermore, the adherence characteristics of newly diagnosed COPD patients were not analyzed in these studies. Therefore, in this prospective study, these limitations are addressed. This study was done by collecting detailed information about the prescriptions and the actual use of medications for COPD and treatment adherence using questionnaires by telephone follow-up, to investigate the causes of adherence and nonadherence to medication in COPD patients in Southwest China. This is the first study on the adherence behavior of newly diagnosed COPD patients and the first large-scale clinical study targeting the Chinese population.

We present the following article in accordance with the MDAR reporting checklist (available at http://dx.doi. org/10.21037/apm-20-1741).

\section{Methods}

Similar to our previous study (10), this study was a prospective study on 24-week telephone follow-up conducted in 12 hospitals in Chongqing, China. The study included one outpatient visit and a total of three telephone follow-ups: 4,12 , and 24 weeks after the outpatient visit. Information on sex, age, history of COPD diagnosis, modified British Medical Research Council (mMRC) dyspnea score, acute exacerbation of COPD in the past year, route of administration, GOLD stage (COPD stage), and distance from the place of permanent residence to the hospital were collected during the outpatient visit. Selfreports related to treatment adherence were collected during the telephone follow-ups, in which detailed information about prescriptions and the actual use of medications for COPD were collected. During the specific time periods before the follow-ups (2 weeks before the 4-week telephone follow-up, 4 weeks before the 12 -week telephone follow-up, and 4 weeks before the 24-week telephone follow-up), the ratios of actual drug consumption to expected drug consumption were used to categorize adherence. A ratio of $80 \%$ to $<120 \%$ was defined as high adherence, and a ratio outside this range was considered nonadherent. The follow-up reports were jointly proposed by the subjects and their guardians, and those who reported nonadherence were asked to choose one of the nine options for potential causes of their nonadherence (see Box 1 below). These options were set up basing on their ease of 


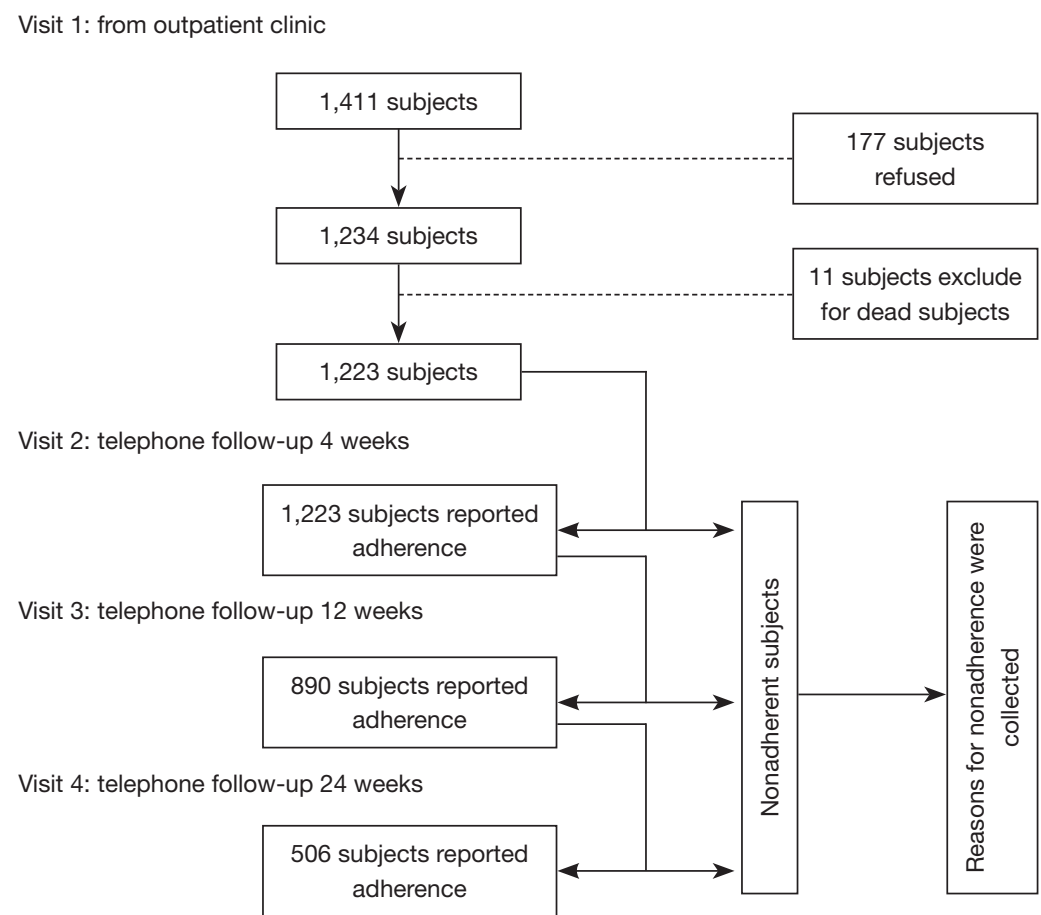

Figure 1 Study design. The schematic shows the study design and the numbers of subjects evaluated at the clinic visit and in the three follow-up telephone calls.

understanding and the World Health Organization (WHO) recommendations (11). The schematic diagram of the study design is shown in Figure 1. This study was approved by the Medical Ethics Committee of Xinqiao Hospital, the Third Military Medical University. Our research passed the review of the ethics IRB committee and reference number is "Clinical review Number 2012012 in Third Military Medical University". All procedures performed in this study involving human participants were in accordance with the Declaration of Helsinki (as revised in 2013). All patients or guardians signed informed consent forms (Registration No: ChiCTR-OCH-12002744). The research data were managed using the clinical database system of the Chronic Airway Disease Management Center of the Department of Respiratory Diseases of Xinqiao Hospital.

\section{Statistical analysis}

SPSS 12 software was used for data analysis. Statistical significance was defined as a $\mathrm{P}$ value less than 0.05 . In the first step, a Cox regression model was used to analyze the relationships between influencing factors of nonadherence and time. In the second step, the chi-squared test was used to compare proportions to analyze the adherence in different age groups, the causes of nonadherence, and their relationships with sex, diagnosis, and age.

\section{Results}

\section{Basic clinical information}

In total, 1,411 patients were screened. After exclusion of 172 patients who refused to participate and five patients who were not prescribed maintenance treatment drugs, 1,234 patients were enrolled in this study. Later, 11 patients who died during telephone follow-up were excluded. Eventually, the data of 1,223 patients, including 965 males and 258 females, were analyzed. There were 929 cases of newly diagnosed COPD and 294 cases of previously diagnosed COPD. The average age of the patients was $61.98 \pm 1.28$ years. More than $60 \%$ of the patients had poor pulmonary function, which was categorized as stage 3 or 4 under the GOLD classification. The distance from the place of permanent residence to hospital was shorter than $80 \mathrm{~km}$ for most patients (Table 1). 
Table 1 Patient characteristics

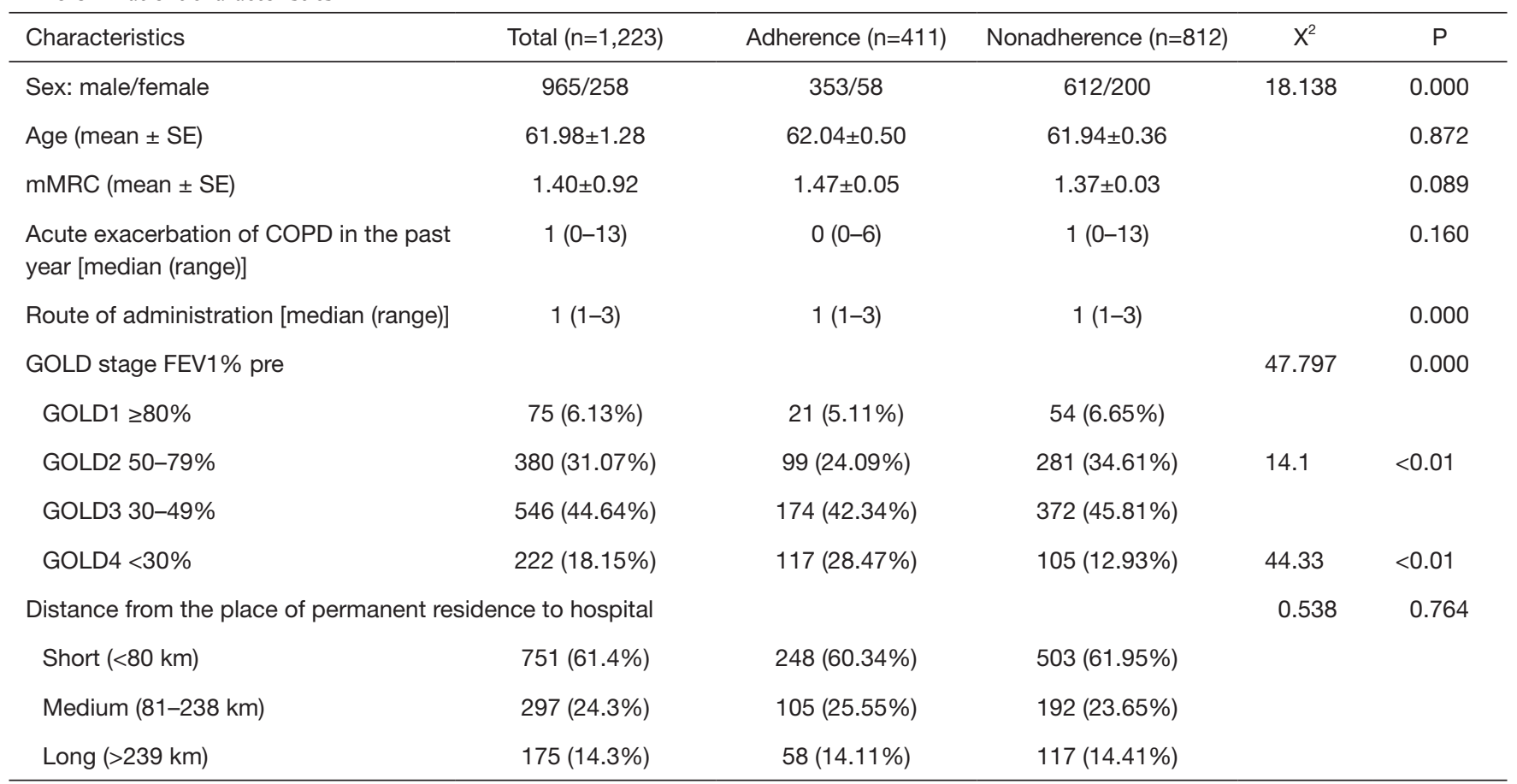

mMRC, modified British Medical Research Council; COPD, chronic obstructive pulmonary disease; GOLD, Chronic Obstructive Lung Disease.

\section{Analysis of treatment adberence and factors influencing treatment adberence}

The adherence rates were $74.65 \%, 49.63 \%$, and $33.61 \%$ at 4, 12, and 24 weeks, respectively. Through analysis using the Cox regression model, sex (Figure $2 A$ ), diagnostic status (newly diagnosed and previously diagnosed) (Figure $2 B$ ), GOLD stage (Figure 2C), and route of administration (Figure 2D) were found to be the four major factors influencing adherence s sex hazard ratio $(\mathrm{HR})=1.39[95 \%$ confidence interval (CI): 1.18-1.63], $\mathrm{P}<0.001$, diagnostic status HR $=1.19(95 \%$ CI: $1.00-1.42), \mathrm{P}<0.001$, COPD stage $\mathrm{HR}=0.86(95 \% \mathrm{CI}: 0.79-0.94), \mathrm{P}=0.001$, route of admission $\mathrm{HR}=0.80$ (95\% CI: 0.69-0.92), $\mathrm{P}=0.002$. The variables included in the analysis were age, sex, diagnostic status, GOLD stage, mMRC dyspnea score, acute exacerbation of COPD in the past year, distance from the place of permanent residence to hospital, and route of administration. Logistic regression was used to analyze highrisk factors affecting nonadherence at 4,12 , and 24 weeks. The results showed that these factors had common features and differences. Sex and GOLD stage affected adherence at 4, 12, and 24 weeks. Female patients [4 weeks, odds ratio $(\mathrm{OR})=1.77,95 \%$ CI: 1.31-2.39; 12 weeks, OR $=1.79,95 \%$
CI: 1.34-2.39; 24 weeks, OR =1.99, 95\% CI: 1.43-2.78] and patients with mild GOLD stage (4 weeks, OR $=0.76,95 \%$ CI: $0.64-0.89 ; 12$ weeks, OR $=0.65,95 \%$ CI: $0.59-0.76$; 24 weeks, OR $=0.76,95 \%$ CI: $0.65-0.89$ ) were more prone to nonadherence. Analysis of diagnostic status showed that patients with newly diagnosed COPD were more prone to nonadherence at 12 and 24 weeks ( 12 weeks, $\mathrm{OR}=1.99$, 95\% CI: $1.49-2.66$; 24 weeks, OR =2.26, 95\% CI: 1.69 3.02). Patients who lived far from the hospital were more prone to nonadherence at 4 weeks ( 4 weeks, OR $=1.19,95 \%$ CI: 1.00-1.42). Patients with fewer routes of administration were more prone to nonadherence at 4 weeks and 24 weeks (4 weeks, OR $=0.63,95 \%$ CI: $0.48-0.83 ; 24$ weeks, OR $=0.63,95 \%$ CI: 0.49-0.79). Details are shown in Table 2 .

\section{Causes of nonadberence}

\section{Overall analysis}

The causes of nonadherence to treatment were analyzed through statistical analysis of the patients included in this study. The results showed that the leading causes of drug nonadherence were drug stoppage after remission (27.2\%), economic factors $(21.5 \%)$, and poor efficacy $(21.1 \%)$. 
A

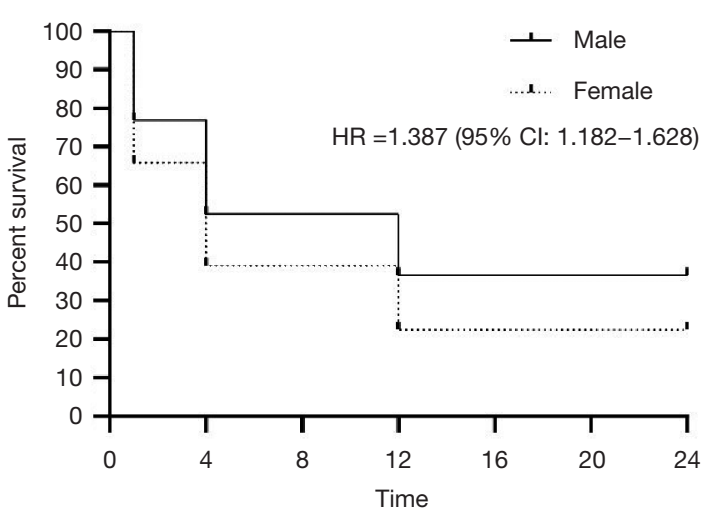

$\mathrm{HR}=0.803(95 \% \mathrm{Cl}: 0.699-0.922)$

C

GOLD Stage

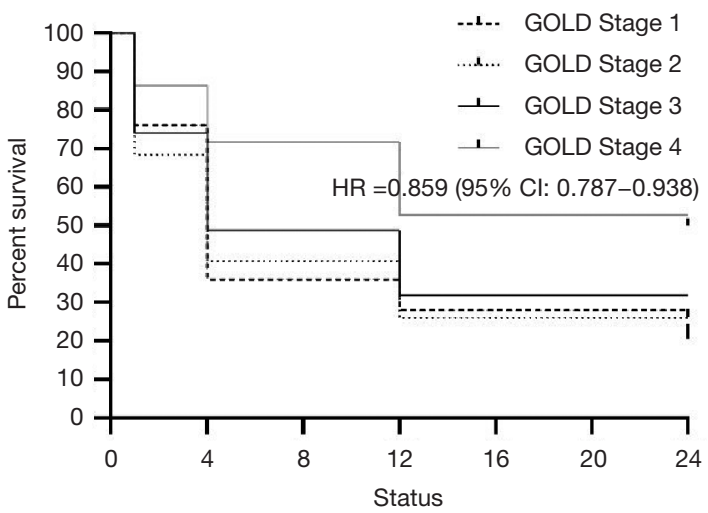

B

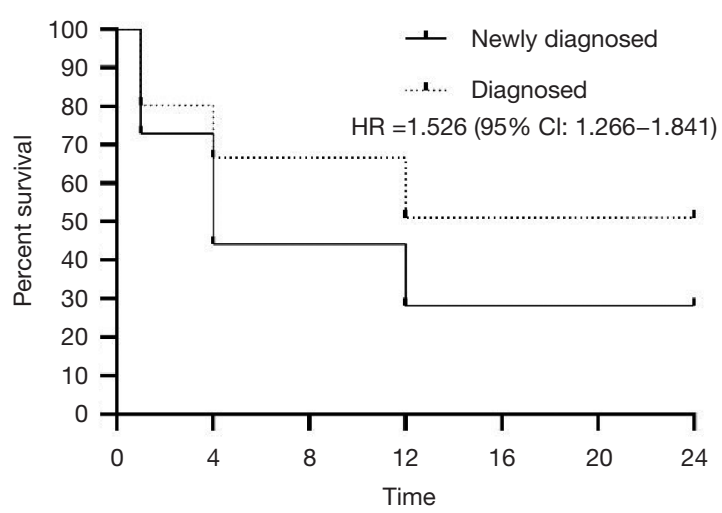

D

Routes of administration

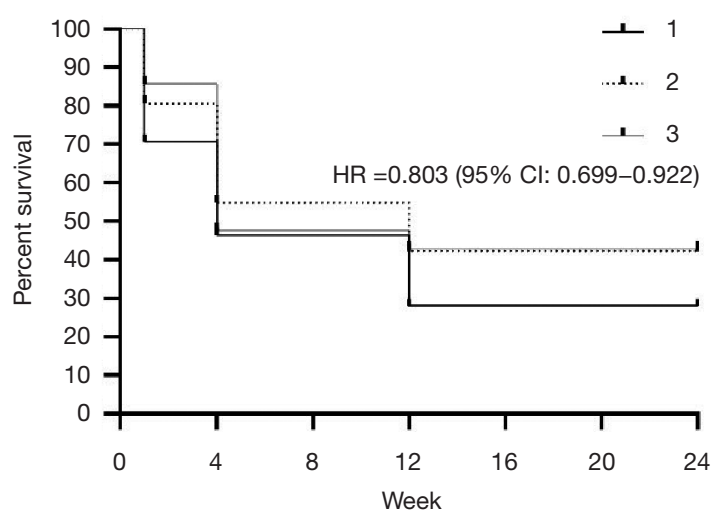

Figure 2 Effects of clinical characteristics on treatment adherence. (A) Self-reported adherence of subjects by Gender. (B) Self-reported adherence of subjects by Diagnosis status. (C) Self-reported adherence of subjects by GOLD stage. (D) Self-reported adherence of subjects by administration route counters. HR, hazard ratio. CI, confidence interval; GOLD, Chronic Obstructive Lung Disease.

The analysis of age showed that the proportion of patients who named economic factors as the cause of nonadherence gradually increased with age $(12.5 \%$ in the $<50$-year-old group, $17.7 \%$ in the $50-59$-year-old group, $23.3 \%$ in the $60-69$-year-old group, $30 \%$ in the $\geq 70$-year-old group, $\mathrm{P}=0.000$ ). The proportion of patients who named inconvenience of treatment gradually increased with age (1\% in the $<50$-year-old, $2.8 \%$ in the 50 -to-59-year-old group, $9.7 \%$ in the 60 -to- 69 -year-old group, $\mathrm{P}<0.01$ ) until slightly decreasing in the $\geq 70$-year-old group (3.6\%). Compared with the $\geq 70$-year-old group, the $<50$-year-old group had a higher rate of drug nonadherence due to poor efficacy $(15 \%$ vs. $27.1 \%, \mathrm{P}=0.022)$ (Figure $3 A$ ).

The comparison of the sexes showed that the proportion of patients who named poor efficacy was higher in females (25.3\% vs. 19.8\%, $\mathrm{P}<0.01)$ (Figure $3 B)$.

Time hierarchical analysis showed that in the 4th week, the proportion of patients who said they stopped taking the drug because of remission was lower $(18.5 \%)$ than in the 12 th week $(32.8 \%)$ or 24 th week $(34.4 \%)$, and the proportion of patients who named the inconvenience of treatment $(9.8 \%)$ was higher than in the 12 th week $(2.5 \%)$ or 24th week $(1.1 \%)(\mathrm{P}<0.01)$ (Figure $3 C)$.

\section{Analysis of different patients grouped by diagnostic status}

In the comparison of groups divided by diagnostic status, patients who said they stopped taking the drug because 
Table 2 Cox regression analysis of the factors affecting adherence

\begin{tabular}{|c|c|c|c|c|c|c|}
\hline & \multicolumn{2}{|c|}{4 weeks } & \multicolumn{2}{|c|}{12 weeks } & \multicolumn{2}{|c|}{24 weeks } \\
\hline Female & 1.77 & $1.31-2.39$ & 1.79 & $1.34-2.39$ & 1.99 & $1.43-2.78$ \\
\hline Newly diagnosed patients & & & 1.99 & $1.49-2.66$ & 2.26 & $1.69-3.02$ \\
\hline Diagnostic status & 1.19 & $1.00-1.42$ & & & & \\
\hline Routes of administration & 0.63 & $0.48-0.83$ & & & 0.63 & $0.49-0.79$ \\
\hline
\end{tabular}

OR, odds ratio; $\mathrm{Cl}$, confidence interval; GOLD, Chronic Obstructive Lung Disease.

of remission accounted for the highest proportion among newly diagnosed cases $(25.5 \%)$ and previously diagnosed cases $(35.2 \%)$. In the newly diagnosed group, the proportion of patients who named concerns about adverse effects as the reason was higher than that in the previously diagnosed group ( $13.6 \%$ vs. $1.85 \%, \mathrm{P}=0.01)$. The proportion of newly diagnosed patients who named concerns about drug resistance was lower than that in the previously diagnosed group $(0.9 \%$ vs. $6.5 \%, \mathrm{P}=0.000)$, as was the proportion who stopped taking the drug after remission $(25.4 \%$ vs. $35.2 \%$, $\mathrm{P}=0.039$ ) (Figure 3D).

In the time hierarchical analysis, except for the presence of the abovementioned overall data differences, the proportions of newly diagnosed patients who named poor efficacy and inconvenience of treatment were higher in the 4th week than in the 12 th week $(27.2 \%$ vs. $17.9 \%, \mathrm{P}=0.002$ and $10.8 \%$ vs. $2.1 \%, \mathrm{P}=0.000$, respectively). The proportion of newly diagnosed patients who named the inconvenience of treatment in the 4th week was also higher than that in the 24 th week $(10.8 \%$ vs. $0, \mathrm{P}<0.004)$. The proportion of newly diagnosed patients who blamed remission was lower in the 4th week $(13.8 \%)$ than in the 12 th week $(32.9 \%)$ or 24th week $(33.8 \%)(\mathrm{P}=0.000)$ (Figure $3 E)$. However, the proportions of patients naming different options in the previously diagnosed group was were not different between time periods (Figure $3 F$ ).

\section{Discussion}

COPD is one of the leading causes of disability and death among chronic diseases worldwide. Under optimal self-management, the physiological and psychological conditions of COPD patients can be improved. Medication is an important aspect of the management of COPD. The risk of death from COPD is significantly higher when pharmacologic management is not initiated or is not sustained (12). Compared with patients with other major chronic diseases, such as heart disease, hypertension, and anxiety and depression, patients with COPD have a lower medication possession rate (13). A study of 244 cases of COPD showed that the rate of adherence to treatment with inhaled corticosteroids combined with LABA was lower than $23 \%$ (14). Although the methods and the standards for the definition of adherence differ between studies, it is not surprising that the adherence was low in our study. The COPD patients in the Chongqing area of China had an adherence rate less than $50 \%$ at the 12 -week follow-up and only $33.61 \%$ at the 24 -week follow-up. Considering that the data were based on self-evaluated survey results, the patients might even have subjectively overestimated their adherence rate (15).

Analysis using the Cox regression model showed that sex, diagnostic status (newly diagnosed and previously diagnosed), GOLD stage, and route of administration all affected adherence. The probability of nonadherence in female patients was $38.7 \%$ higher $(\mathrm{HR}=1.387)$ than that in male patients, and female patients were more likely to be nonadherent than male patients at all evaluation time points. This result is similar to previous findings on chronic respiratory diseases (6), suggesting that sex affects adherence in COPD patients, and more attention needs to be paid to sex differences.

Our study gives us the opportunity to distinguish for the first time the differences in medication adherence between newly diagnosed and previously diagnosed COPD patients. The likelihood of nonadherence in newly diagnosed COPD patients was more than $50 \%$ higher than that in the previously diagnosed patients $(\mathrm{HR}=1.526)$. Nonadherence tended to be observed more in the 12th and 24th weeks. The main cause of nonadherence was stoppage after 

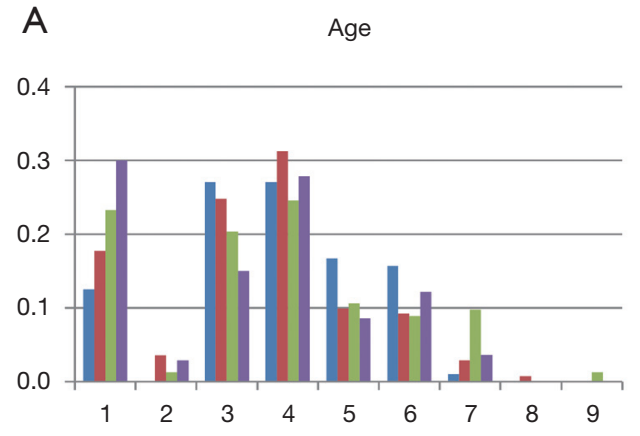

\section{C}

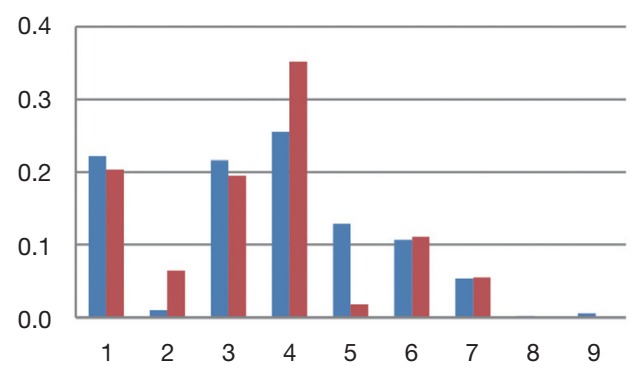

E

Time-causes analysis in newly diagnosed patients

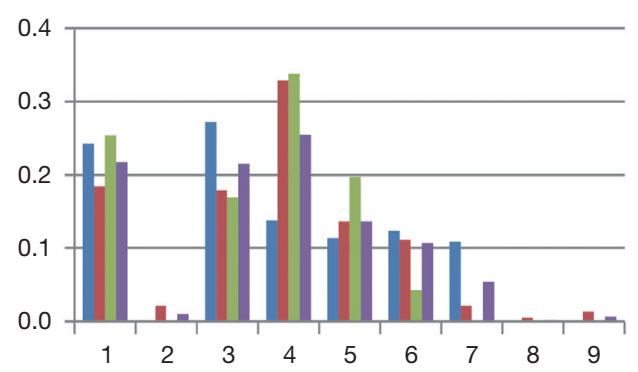

B

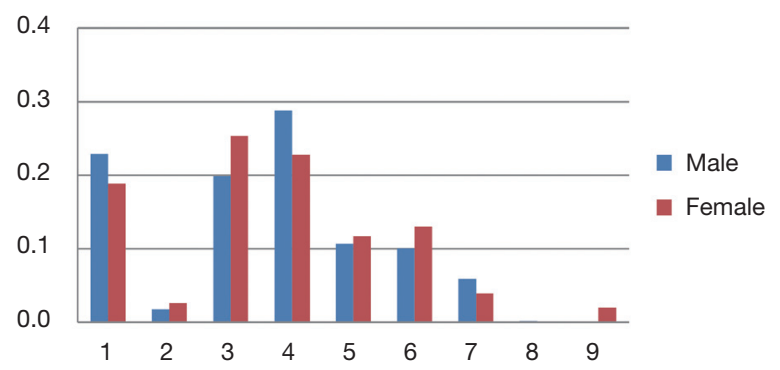

D Time-causes

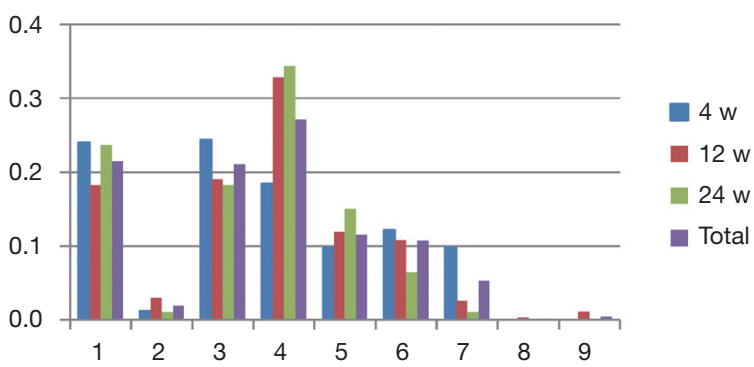

F Time-causes analysis in previously diagnosed patients

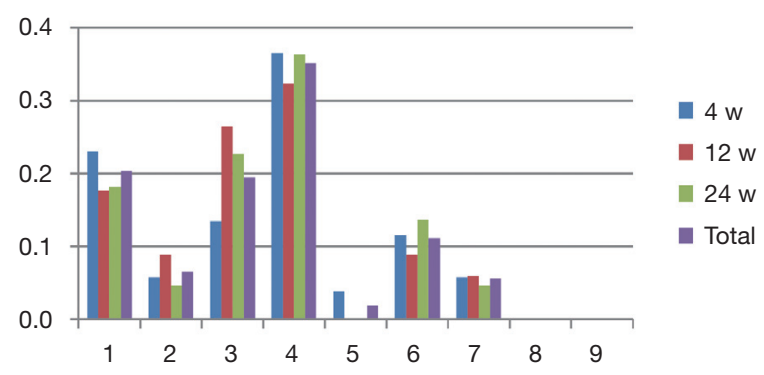

Figure 3 Causes of treatment non-adherence: No. 1 high cost of medication, No. 2 diminished treatment effectiveness with long-term use, No. 3 poor self-efficacy, No. 4 relief of symptoms after short-term controlled medication use, No. 5 occurrence/fear of side effects, No. 6 difficulty obtaining medication, No. 7 inconvenience of treatment, No. 8 preference for oral medication, No. 9 other. (A) Comparison of total self-reported causes of non-adherence between various age groups. (B) Comparison of total self-reported causes of non-adherence between males and females. (C) Self-reported causes of non-adherence at each of the three follow-up phone calls and in total. (D) Comparison of total self-reported causes of non-adherence between newly diagnosed patients and patients with a history of COPD. (E) Selfreported causes of non-adherence at each of the three follow-up phone calls and in newly diagnosed patients. (F) Self-reported causes of non-adherence at each of the three follow-up phone calls and in patients with a history of COPD. COPD, chronic obstructive pulmonary disease.

remission, and the proportion of patients who named this as a reason accounted for approximately $1 / 3$ of all nonadherent patients. We recommend strengthening the education of COPD patients, especially newly diagnosed patients, and to inform patients that COPD is a chronic disease that requires long-term management and that, after remission, consultation with professional physicians instead of selfevaluation is needed to decide when to stop medication. 
More measures should be taken to improve the education of patients to avoid exacerbation events and other treatment events.

Patients with high GOLD stages tended to be adherent throughout the study period, and no significant difference was observed in the mMRC dyspnea score between the newly diagnosed and previously diagnosed patients. Adherence in COPD patients has shown no significant correlation with relief or the severity of symptoms (8). Not only should the means of treatment and intervention meet a certain standard, but also the burden (including pain and the costs of intervention, such as money and energy) put on patients by COPD treatments should be evaluated so that the intervention has less of a negative effect than the disease does. The cost-to-effectiveness ratio of the disease management process should be comprehensively evaluated.

It is generally believed that a more complexity of drug administration reduces medication adherence. For example, a higher number of inhaler devices for delivering inhaled COPD medications reduces adherence (16). In recent years, manufacturers of drugs have tended to put a variety of drugs into a single device while cutting down the number of drug administrations from two times per day to one time per day, thereby reducing the complexity of drug administration. However, our results showed that the fewer the routes of administration, the higher the likelihood of nonadherence in the 4th and 24th weeks. According to our understanding, in the short term (4 weeks), having more routes of administration (e.g., symptomatic treatment such as antitussive and expectorant treatment) more successfully relieved the symptoms or enhanced the sense of ritual and thus improved adherence. In the middle term (12 weeks), patients tended to stop medication due to tradeoffs between cost and effectiveness. In the long term (24 weeks), most patients were satisfied with their symptom control and were able to accept the costs considering the effectiveness of the medication, while some patients had to comply with longterm treatment due to relapse of symptoms after shortterm drug stoppage, even though they did not report the temporary stoppage.

It is generally believed that the further the patient resides from the hospital, the more difficult it is to obtain medical resources, and the poorer the adherence. This study found that patients who were lived over $80 \mathrm{~km}$ away from the hospital were more inclined to nonadherence in the 4th week, but this trend was not observed in the 12 -week follow-up. The primary goal of these patients in the initial diagnosis was to clarify the diagnosis and then select the nearest medical facility that could treat them. This might have been related to the limited service the patients could receive from local medical facilities due to their low capacity. Low service capacity remains a challenge for the nearest medical facility to provide the continuous medical treatment. In practice, some patients may even give up treatment after the initial diagnosis of COPD. Therefore, when developing management plans for patients who live far from the hospital, especially for patients diagnosed within the last 4 weeks, it is necessary to consider these factors to enhance their adherence. At the same time, in the health care system, cross-regional sharing of patient information and communication between medical facilities at all levels should be achieved to realize the whole-course management of COPD.

The main purpose of this study was to further analyze the main causes of nonadherence in COPD patients and to lay the foundation for targeted interventions. The main causes of nonadherence included remission of the disease, economic factors, and poor efficacy. Nonadherence due to disease remission accounted for nearly $1 / 3$ of all-cause nonadherence and was mainly concentrated in the 12 th and 24th weeks. First, adherence was analyzed based on drug stoppage time. Due to the constraints of medical insurance policies and inhaler devices, patients received a 1-month supply of conventional prescription drugs for COPD at first in the clinic. Therefore, the supply should have lasted through the 4-week follow-up. Some patients might adhere to the medication simply to avoid wasting the drugs because they still have some left. This might lead to an overestimation of the adherence rate. Second, adherence was analyzed from the perspective of causes of nonadherence. Although no correlation between symptom relief and medication adherence was found, we still found that after clinical remission, patients tended to stop medication due to the subjective feeling of improvement. The questionnaires reflected the patients' understanding of COPD, their self-evaluation of the severity of their COPD, and the need for treatment mainly based on indicators such as symptoms and limitation of motion. There is no reliable efficacy feedback mechanism to keep track of and maintain medication adherence. In addition, due to the poor understanding of the necessity of long-term medication for chronic disease, COPD patients have a lower medication possession rate than patients with other chronic diseases, such as hypertension and heart diseases. Stronger patient education may improve these conditions. Although there are no studies on the impact of education on the adherence 
in COPD patients, a Canadian study (17) has shown that individualized education can promote the use of medications in COPD patients. The education of outpatients has not been fully implemented in most hospitals in China due to restrictions by influencing factors such as clinic size and the time of the visit. Therefore, strengthening the necessary patient education may alleviate the high nonadherence rate in this region.

Economic factors were the second leading cause of nonadherence, including the cost-to-effectiveness ratio of self-management of COPD, as mentioned above, and the actual drug expenditure. The average per capita income in Chongqing is 6,106 yuan/month (approximately 863 dollars), and the per capita income after retirement decreases to less than 3,000 yuan/month (approximately 424 dollars). Therefore, retired COPD patients are more sensitive to the medication costs. The average monthly medication expense of COPD patients is approximately 400 yuan (approximately 57 dollars), accounting for 13.3\% of the postretirement income, and 91\% of COPD patients in Chongqing are covered by health insurance. After reimbursement, the actual monthly average expenditure on medication is approximately 162 yuan (23 dollars), accounting for $5.4 \%$ of postretirement income. It can be expected that the proportions of nonadherent patients due to economic factors will be higher in patients with low income or without medical insurance. Increasing postretirement income, lowering drug prices, and optimizing health care strategies can help solve this problem.

Poor efficacy was the third leading cause of nonadherence. The meaning of efficacy here is significantly different from the meaning of clinical efficacy in routine clinical studies. The indicators for evaluating traditional clinical efficacy mainly include pulmonary function indices, quality of life based on patient-reported outcomes, number of acute exacerbations, death, etc. The efficacy in this study better reflects whether the degree of relief of symptoms, including the limitation of motion, met the patient's expectations after receiving the treatment. In the $\geq 70$-year-old group, the proportion of patients who stopped medication due to poor efficacy was lower than that in the $<50$-year-old group. Since the patients younger than 50 years old were still working, they might simply have chosen the option of poor efficacy because the desired efficacy was not achieved because they stopped taking the drug to avoid the negative effects of long-term medications on their careers.

The that the proportion of patients who named inconvenience as the reason for treatment nonadherence increased with age among patients aged $<70$ years might be related to factors such as a decreasing ability to move and a decreasing ability to operate the inhaler device. However, this proportion decreased in patients aged $\geq 70$. Previous studies showed that in elderly people, a higher degree of self-reliance was associated with poorer medication adherence (18). This might suggest that compared with other elderly patients, self-reliance was poorer in our patients aged $\geq 70$ years. However, their younger relatives helped them get the medicine home, brought them to the follow-up, and supervised their taking of medicine on time, thus reducing the occurrence of drug stoppage due to the inconvenience of treatment. Our study also found that the occurrence of drug stoppage due to inconvenience of treatment was the highest in the 4th week, suggesting that it would increase the adherence within 4 weeks for newly diagnosed patients if this issue could be addressed.

According to the subgroup analysis of the diagnostic status, the overall adherence in newly diagnosed patients was poorer than that in previously diagnosed patients. The former had lower acceptance of the treatment expense, higher expectations about its efficacy, and more concerns about the side effects, suggesting that strengthening the COPD-related education for newly diagnosed patients before treatment is conducive to improving adherence.

Negative emotions such as depression may be related to COPD patients' non-compliance. A logistic regression analysis showed that depression was independently associated with non-compliance (19). In addition, indicators including pulmonary rehabilitation and health-related quality of life might also be related to non-compliance. Because this study is a prospective design, the main goal is to achieve the causes of non-compliance. For example by telephone interviews it's very difficult to obtain relatively scientific data about quality of life and recurrence rate. We have to give up the collection of these data in the research design, and we look forward to improving it in more research.

\section{Conclusions}

COPD patients in Chongqing had poor adherence to medication, with an adherence rate of $33.61 \%$ at the 24-week follow-up. Nonadherent patients tended to be female, newly diagnosed patients, patients with mild GOLD stage, and patients with fewer routes of administration. The leading causes of nonadherence were disease remission, 
economic factors, and poor efficacy.

Our results suggest that adherence is not an all-or-none phenomenon but rather a series of dynamic behaviors. At different time points, the patient's demands, satisfaction levels, and commitment to disease management (target) also differ. Therefore, in addition to balancing medical measures, meeting the needs of patients at different time points is critical to improving adherence.

The results of this study suggest that adherence can be improved by expanding the capacity and accessibility of medical clinics, detecting early those patients with a high risk of nonadherence, finding the specific causes of nonadherence in individual patients and conducting targeted interventions, and improving the convenience and accessibility of treatment.

\section{Acknowledgments}

Funding: None.

\section{Footnote}

Reporting Checklist: The authors have completed the MDAR reporting checklist. Available at http://dx.doi.org/10.21037/ apm-20-1741

Data Sharing Statement: Available at http://dx.doi. org/10.21037/apm-20-1741

Conflicts of Interest: All authors have completed the ICMJE uniform disclosure form (available at http://dx.doi. org/10.21037/apm-20-1741). The authors have no conflicts of interest to declare.

Ethical Statement: The authors are accountable for all aspects of the work in ensuring that questions related to the accuracy or integrity of any part of the work are appropriately investigated and resolved. The study was approved by the Medical Ethics Committee of Xinqiao Hospital, the Third Military Medical University. Our research passed the review of the ethics IRB committee and reference number is "Clinical review Number 2012012 in Third Military Medical University". All procedures performed in this study involving human participants were in accordance with the Declaration of Helsinki (as revised in 2013). All patients or guardians signed informed consent forms (Registration No: ChiCTR-OCH-12002744).
Open Access Statement: This is an Open Access article distributed in accordance with the Creative Commons Attribution-NonCommercial-NoDerivs 4.0 International License (CC BY-NC-ND 4.0), which permits the noncommercial replication and distribution of the article with the strict proviso that no changes or edits are made and the original work is properly cited (including links to both the formal publication through the relevant DOI and the license). See: https://creativecommons.org/licenses/by-nc-nd/4.0/.

\section{References}

1. Wang C, Xu J, Yang L, et al. Prevalence and risk factors of chronic obstructive pulmonary disease in China (the China Pulmonary Health [CPH] study): a national cross-sectional study. Lancet 2018;391:1706-17.

2. Kaplan RM, Ries AL. Quality of life as an outcome measure in pulmonary diseases. J Cardiopulm Rehabil 2005;25:321-31.

3. Ko FW, Chan KP, Hui DS. Comprehensive care for chronic obstructive pulmonary disease. J Thorac Dis 2019;11:S2181-91.

4. Bender BG. Nonadherence in chronic obstructive pulmonary disease patients: what do we know and what should we do next? Curr Opin Pulm Med 2014;20:132-7.

5. Simoni-Wastila L, Wei YJ, Qian J, et al. Association of chronic obstructive pulmonary disease maintenance medication adherence with all-cause hospitalization and spending in a Medicare population. Am J Geriatr Pharmacother 2012;10:201-10.

6. Mueller S, Wilke T, Bechtel B, et al. Non-persistence and non-adherence to long-acting COPD medication therapy: A retrospective cohort study based on a large German claims dataset. Respir Med 2017;122:1-11.

7. George M. Adherence in Asthma and COPD: New Strategies for an Old Problem. Respir Care 2018;63:818-31.

8. Duarte-de-Araújo A, Teixeira P, Hespanhol V, et al. COPD: understanding patients' adherence to inhaled medications. Int J Chron Obstruct Pulmon Dis 2018;13:2767-73.

9. George J, Kong DC, Thoman R, et al. Factors associated with medication nonadherence in patients with COPD. Chest 2005;128:3198-204.

10. Ma Q, Luo G, Zhou X, et al. Self-reported reasons for treatment non-adherence in Chinese asthma patients: A 24-week prospective telephone follow-up study. Clin Respir J 2018;12:262-8. 
11. Sabaté E, Sabaté E. Adherence to long-term therapies: evidence for action. World Health Organization; 2003.

12. Mäkelä MJ, Backer V, Hedegaard M, et al. Adherence to inhaled therapies, health outcomes and costs in patients with asthma and COPD. Respir Med 2013;107:1481-90.

13. Yang B, Choi H, Lim JH, et al. The disease burden of bronchiectasis in comparison with chronic obstructive pulmonary disease: a national database study in Korea. Ann Transl Med 2019;7:770.

14. Sulaiman I, Cushen B, Greene G, et al. Objective Assessment of Adherence to Inhalers by Patients with Chronic Obstructive Pulmonary Disease. Am J Respir Crit Care Med 2017;195:1333-43.

15. Palmieri JJ, Stern TA. Lies in the doctor-patient relationship. Prim Care Companion J Clin Psychiatry 2009;11:163-8.

Cite this article as: Zhang M, Tang T, Wan M, Zhang Q, Wang C, Ma Q. Self-reported reasons for treatment nonadherence in chronic obstructive pulmonary disease (COPD) patients: a 24-week prospective cohort study in China. Ann Palliat Med 2020;9(5):3495-3505. doi: 10.21037/ apm-20-1741
16. Yu AP, Guérin A, Ponce de Leon D, et al. Therapy persistence and adherence in patients with chronic obstructive pulmonary disease: multiple versus single longacting maintenance inhalers. J Med Econ 2011;14:486-96.

17. Sari N, Osman M. The effects of patient education programs on medication use among asthma and COPD patients: a propensity score matching with a differencein-difference regression approach. BMC Health Serv Res 2015;15:332.

18. Insel KC, Reminger SL, Hsiao CP. The negative association of independent personality and medication adherence. J Aging Health 2006;18:407-18.

19. Khdour MR, Hawwa AF, Kidney JC, et al. Potential risk factors for medication non-adherence in patients with chronic obstructive pulmonary disease (COPD). Eur J Clin Pharmacol 2012;68:1365-73. 